\title{
Temporary Anchorage Device as an Innovative Alternative for Unilateral Arch Mesialization in Medical Application
}

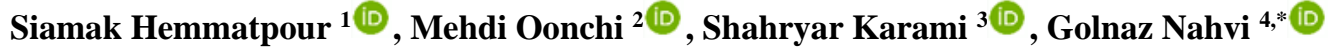 \\ Assistant professor, Department of Orthodontics, School of dentistry, Islamic Azad University, Tehran, Iran \\ Faculty of Dentistry, University of British Columbia, Canada \\ Postgraduate student of Orthodontics, Department of Orthodontics, School of dentistry, Islamic Azad University, Tehran, \\ Iran \\ 4 Department of Orthodontics, School of dentistry, Shahid Beheshti University of Medical Sciences, Tehran, Iran \\ * Correspondence: golnaznahvi@gmail.com;
}

Scopus Author ID 56082419000

Received: 16.07.2020; Revised: 17.08.2020; Accepted: 18.08.2020; Published: 21.08.2020

\begin{abstract}
Like any other medical intervention, orthodontic treatment has, in addition to its benefits, also associated risks and complications that are sometimes caused by wrongful treatment objectives. Correction of previous orthodontic malpractice is always a challenging problem in orthodontics. The present study aimed to introduce an innovative approach for total arch mesialization. In this study, the treatment course of a 25-year-old woman dissatisfied with her previous improper orthodontic treatment plan and unnecessary extraction of the upper and lower right first premolar teeth. Maxillary and mandibular dental midline deviation was evident concurrent with asymmetric dental arches and excessive gingival display a smile. The proposed corrective treatment plan consisted of right side temporary anchorage devices assisted total arch mesialization in both maxilla and mandible, followed by orthognathic surgery at the end. The patient's final occlusion and facial profile improved significantly after the second treatment. The result was determined to be stable over a 1-year period. No significant evidence of root resorption was detected. Overbite decreased from $95 \%$ to $25 \%$. Yaw correction was completely accomplished, and the final lateral cephalometric analysis after orthognathic surgery showed skeletal cl I relationship, normal proclination of upper and lower incisor teeth, and no evidence of periodontal complication and loss of vitality in the mandibular and maxillary teeth. The unilateral mesialization approach used in the current study prevented invasive therapies such as dental implants employing a special approach, which is less aggressive and more durable for the patient and revealed successful results with minimum side effects.
\end{abstract}

Keywords: orthodontics; asymmetry; dental arch.

(C) 2020 by the authors. This article is an open-access article distributed under the terms and conditions of the Creative Commons Attribution (CC BY) license (https://creativecommons.org/licenses/by/4.0/).

\section{Introduction}

Incorrect treatment planning is a relatively common complication in orthodontics. This is true for performing unilateral extractions incorrectly, which not only causes midline shift but also results in dental arches asymmetry, patient's aesthetic dissatisfaction and posterior displacement of surrounding soft tissues [1-6] and also, the oral disease (microbial, cancer, etc.) should be considered beside this treatment [7-10]. There are two orthodontic treatment strategies to resolve this problem. First, in the areas of incorrectly extracted teeth, space should be regained, and subsequently, dental implants should be inserted. Another therapeutic option 
is the total arch mesialization (TAM) in the extraction quadrant. TAM potentially eliminates the need for space reopening for surgical implant insertion and possible bone grafting [11-13], restoration, and all its associated costs and, therefore, can be the treatment or regeneration of choice in some studies $[11,14]$.

Application of temporary anchorage devices (TADs) as skeletal anchorage provides absolute anchorage with rapid and predictable treatment results [11]. For dental arch mesialization, inter radicular mini-screws could be applied. The disadvantages of this type of mesialization include: limited amounts of tooth movement, probability of contacting tooth and periodontal ligament (PDL) damage during screw placement, and further need to reposition of the mini-screws after few millimeters of dental mesialization movement has achieved [15]. To overcome these problems and to accelerate accomplishing the ideal results, total arch mesialization can be accomplished by using a novel TAD-based jig in the area out of the teeth movement path. This study aimed to re-treatment of a patient with a history of unilateral extraction of upper and lower first premolars with upper and lower total arch mesialization. The benefit system was used for maxilla and an innovative TAD-based jig in the buccal shelf area of the mandible specifically designed for unilateral total arch mesialization in the lower arch.

\section{Materials and Methods}

\subsection{Diagnosis.}

A 25-year-old woman referred with the chief complaints of an excessive gingival display during a smile, especially in the right side, increased lower third facial height, and deviation of the upper and lower midlines to the right because of the extraction of the upper and lower right premolars in previous improper orthodontic treatment causing upper and lower dental arches yaw (Figure 1).
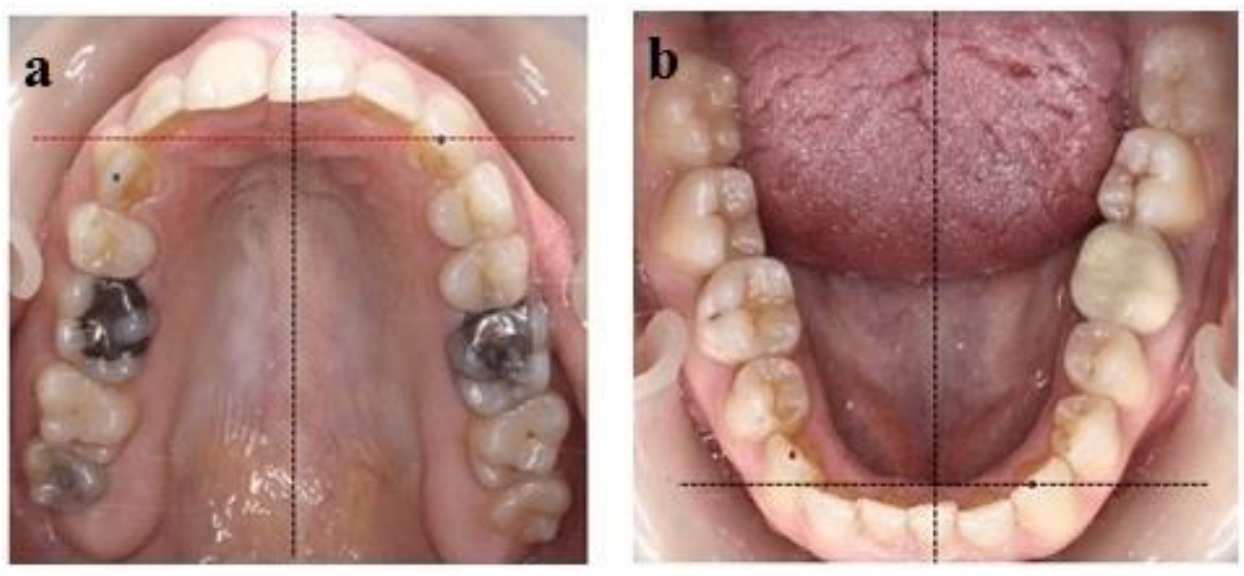

Figure 1. (a) Occlusal view of the upper and (b) lower dental arches yaw rotation.

Due to vertical maxillary excess (VME), her gingival display at rest and smile was excessive. The patient's lips were incompetent. Roll of Maxillary occlusal plan during smile (right side down) was obvious. The patient's overjet was approximately normal, and the overbite was increased (Figures 2 and 3a). The initial lateral cephalometric analysis showed a mild skeletal cl II and retroclination of upper and lower incisors. (Figure $3 \mathrm{~b}$ and Table 1). 


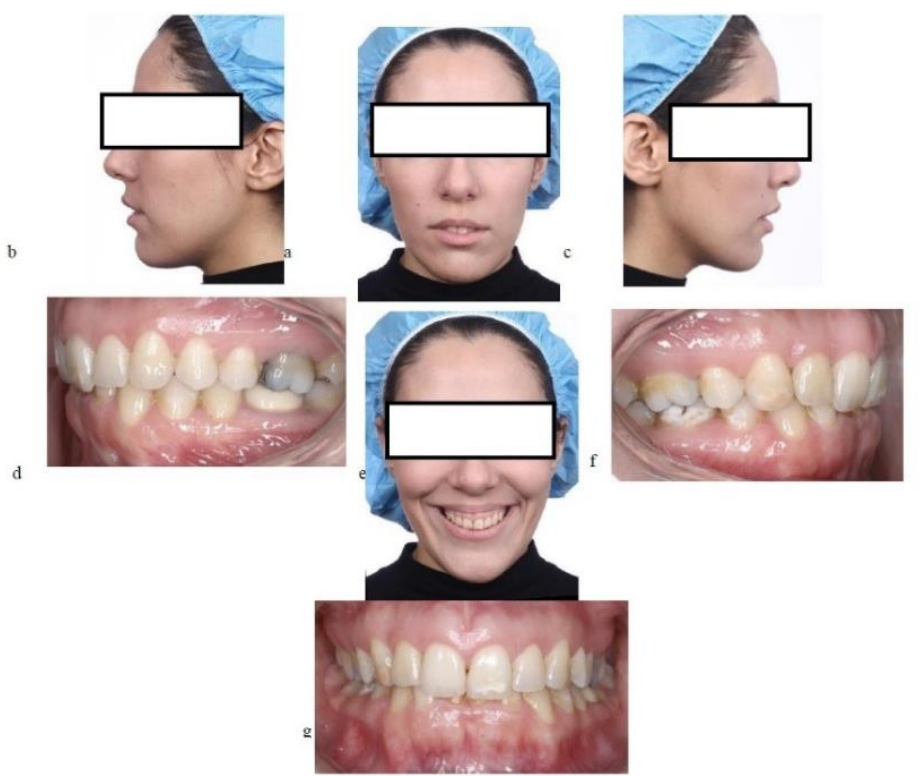

Figure 2. Extraoral views: (a) frontal at rest, (b) left profile and (c) right profile; (d) frontal at smile; intraoral views: (e) left, (f) right, (g) frontal.

a

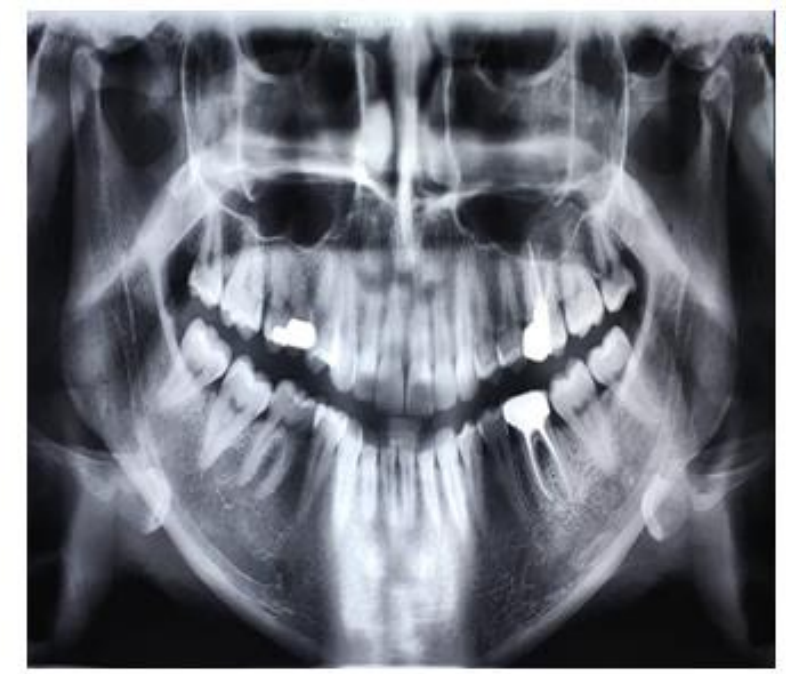

b

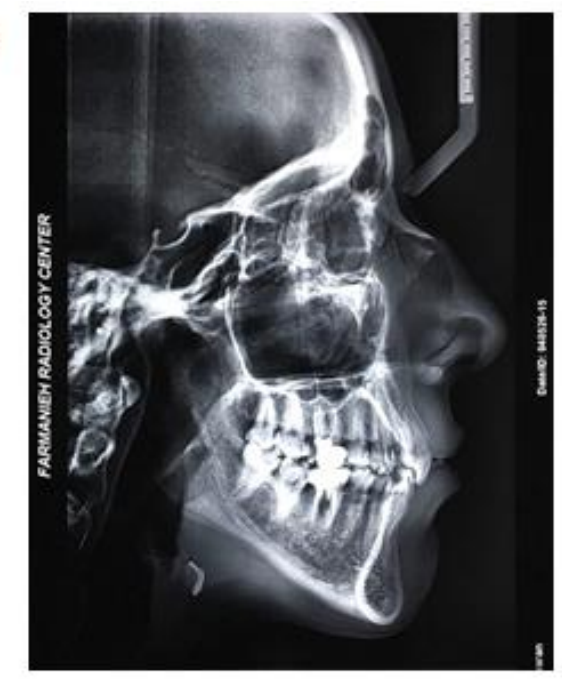

Figure 3. (a) Initial Panoramic view; (b) Initial lateral cephalogram.

Table 1. Pre-treatment values of cephalometric analysis.

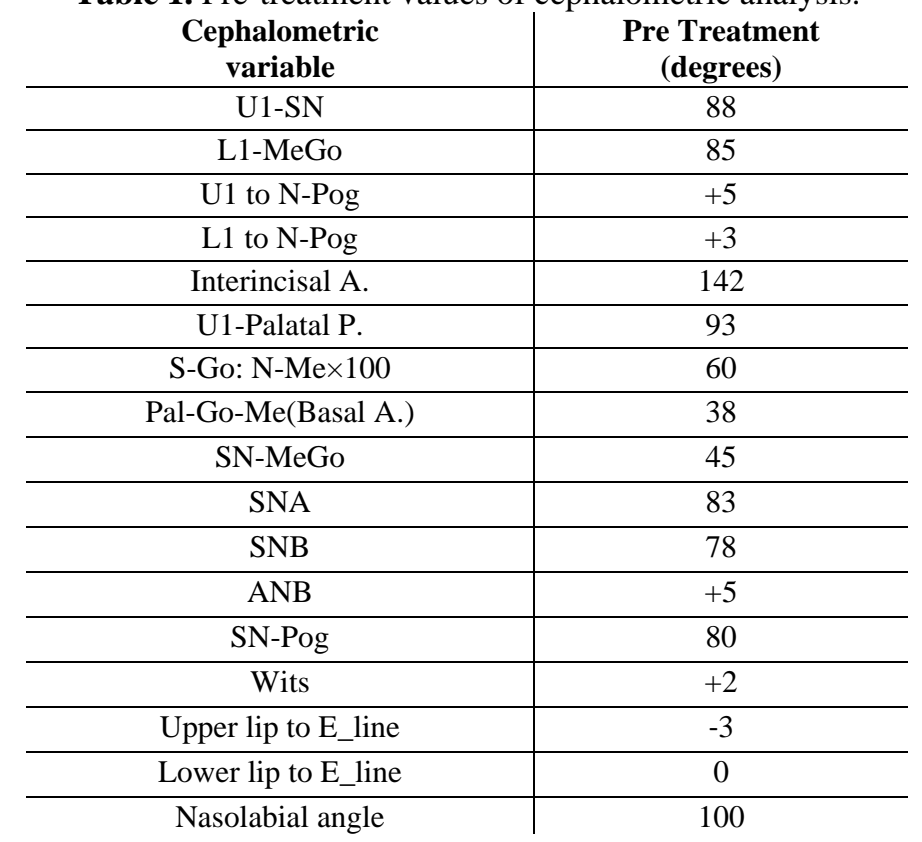




\subsection{Treatment objectives.}

The treatment objectives consisted of improvement of the patient's profile, correction of the upper and lower dental arch midline deviation, improvement of the incisors and gingival show during a smile, achieving normal overbite and overjet, achieving a class I molar and canine relationship, and vertical maxillary excess (VME) orthognathic correction.

\subsection{Treatment alternatives.}

The first treatment option was to space reopening for the right side dental implants, which was declined by the patient. The second option was the extraction of the left upper and lowered first premolars, which were not appropriate in this patient due to her wide buccal corridor, retroclined upper and lower incisors, and the soft tissue profile. The third treatment option was to apply mini-screws in the radicular space of canine and second premolar area to mesialize the posterior teeth. After discussing the pros and cons of this option with the patient and considering the limitation of mesialization amount, probability of damage to the root during mesialization, and the need to replace the mini screw during the treatment, this option was also declined by the patient. The last option was to utilize 2 mini implants in the mid palatal area and another mini implant on the lower right side of the arch distal to the last molar for total mesialization of the arch without the need to reposition the mini screw during the orthodontic treatment. Since this patient also had increased lower facial height, excessive tooth/gingiva show in the upper arch, increased gonial angle, and downward rotation of the mandible, we also recommended orthognathic surgery to address these issues.

\subsection{Treatment progress.}

Prior to the orthodontic treatment, all dental caries were removed and restored. The patient was also referred to an Endodontist to confirm the health of the existing root canal treatments on the upper and lower left first molars. The upper right third molar was extracted by an Oral and Maxillofacial surgeon due to severe tooth decay. At the first orthodontic bonding appointment, only the maxillary arch was bonded. Pre-adjusted MBT metallic brackets (Master Series, American Orthodontics Ltd, Sheboygan, Wis, USA) with 0.022 slot size were utilized. 0.014" superelastic nickel-titanium wire (austenitic active, preformed ovoid, superelastic archwire; 3M Unitek Corporation) was employed for initial alignment and leveling. At subsequent visits, archwire was changed to $0.16 " \mathrm{NiTi}, .018 " \mathrm{NiTi}$ and then $.019 \times .025 "$ rectangular $(\mathrm{NiTi})$ until we could place $19 \times 25 "$ stainless steel as the working archwire.

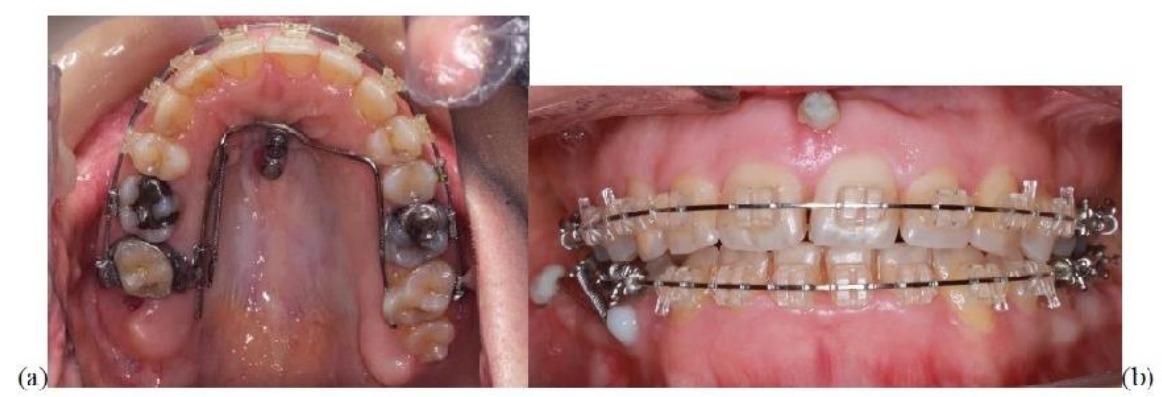

Figure 4. (a) Beneplate inserted in the upper arch; (b) Mini-screw inserted in the midline of the upper jaw to correct the deep bite. 
Two Benefit mini-implants were placed in the anterior palatal area. Immediately after the placement of the mini implants, an impression was taken to adapt the Beneslider on the plaster working models. Incognito palatal sheaths were used for a stable connection to the molar bands. A Beneplate with an attached .043" wire was adapted to the palatal slope, and the Beneslider was placed and activated with a $500 \mathrm{~g}$ nickel-titanium open-coil spring on the right side. The left side of the appliance was left passive. To minimize rocking the device, Beneplate was connected to the second molar with a flowable composite. Another mini-screw $(1.6 \mathrm{~mm} \times 8$ $\mathrm{mm}$ ) was inserted in the midline of the upper jaw between the first central incisors on the labial side to correct the deep bite (Figure 4).

After correcting the deep bite in the initial sequence of the treatment to accommodate the lower brackets, brackets were placed on the mandibular arch to start the leveling and alignment using 0.014-inch NiTi archwire and archwire sequentially changed at the following appointments to accommodate the $19 \times 25$ " stainless steel working archwire. A mini-screw $(1.6 \times 8 \mathrm{~mm}$, JB, Dual Top Anchor System; Jeil Medical, Seoul, Korea) was inserted on the right side, in the distal area of the buccal shelf. A special custom-made jig was designed using a $19 \times 25$ " stainless steel wire (Figure 5). In the posterior area, the jig was connected to the mini-screws and the archwire in the area between the second premolar and first molar.

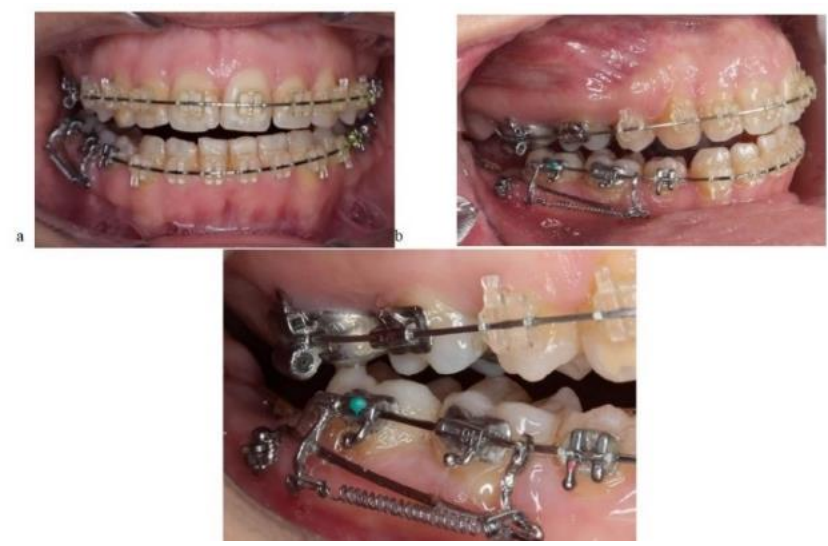

Figure 5. Special type of jig designed for posterior lower right Mesialization (a, b and c).

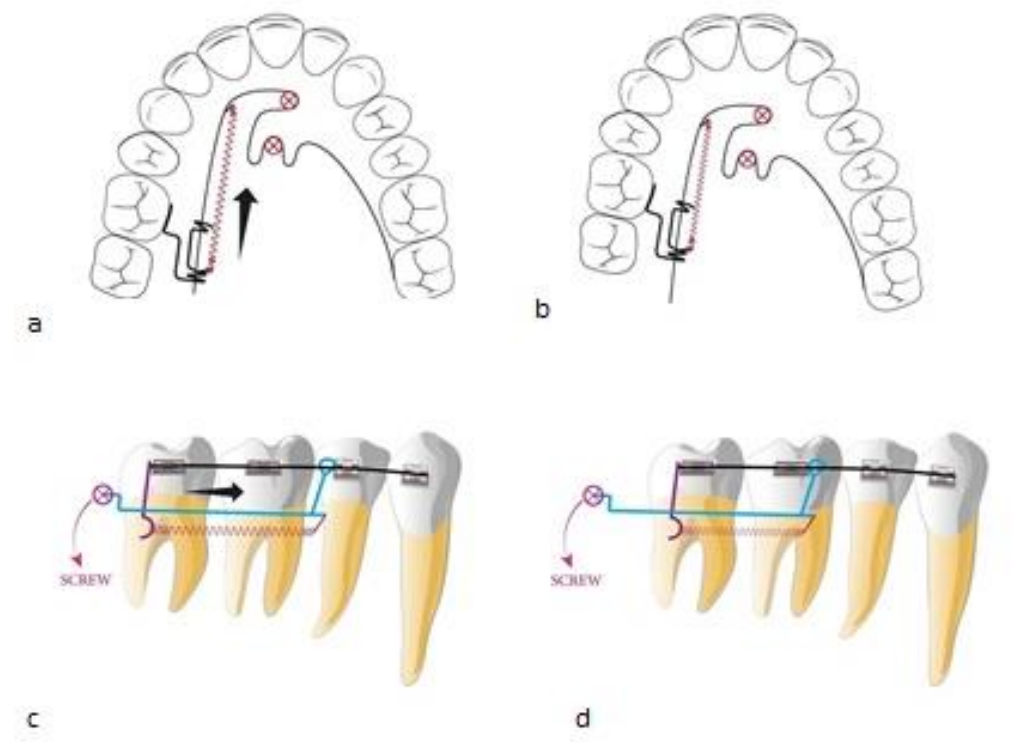

Figure 6. Schematic view of the upper and lower dental arches mesialization. 
Then, a loop was inserted in that region and bent upward and hooked on the archwire in the area between the second premolar and first molar to prevent upward and downward movement of the sliding jig. By using a crimpable hook-shaped lever arm crimped on the wire distal to the lower right second molar, mesialization commenced using a $10 \mathrm{~mm}$ Sentalloy closed coil spring (Dentsply GAC Int., Bohemia, NY, USA) with heavy forced from lever arch to the jig hook (Figure 5). Throughout the treatment time, Beneplate and working wire adjustments were performed, where needed to maintain the upper and lower arches coordination. Dental arches preparation was accomplished in 10 months (Figure 6). At the end of the presurgical orthodontic phase, due to the VME of the patient, a shallow overbite was evident (Figure 7). After surgery over the finishing and detailing stage, final adjustments were performed by vertical elastic. For retention, we used a bonded lingual retainer for six lower anterior teeth, and a Hawley retainer was used for the maxilla. The total duration of treatment was 28 months.

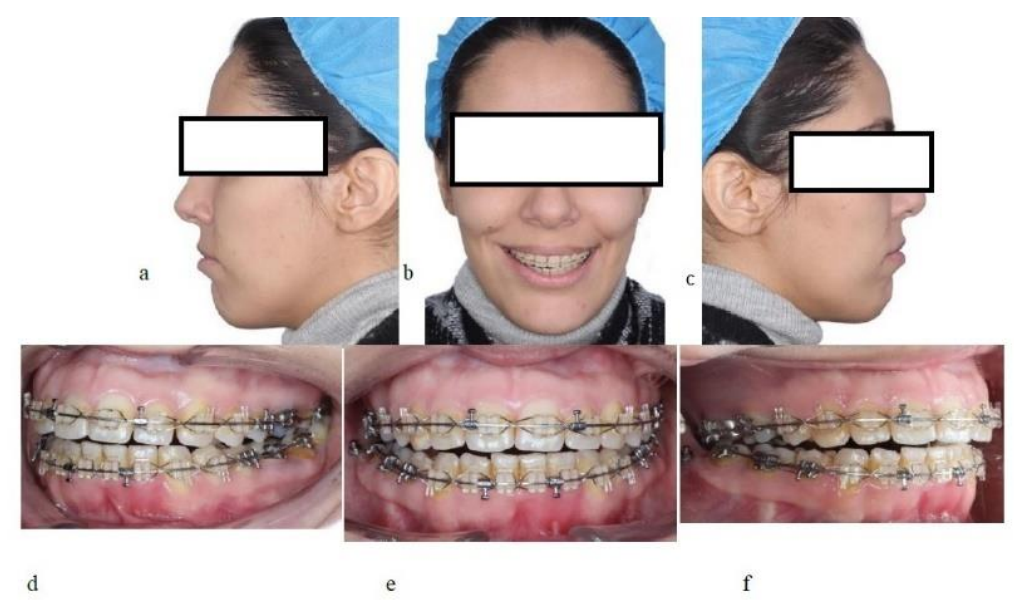

Figure 7. At the end of the presurgical orthodontic phase, extraoral views: (a) left, (b) frontal, (c) right; intraoral views: (d) left, (e) frontal, (f) right.

\section{Results and discussion}

\subsection{Dental and soft tissue findings.}

Great improvement of the incisors and gingival show was evident in the post-treatment phase, and the patient's facial esthetic and profile were improved.

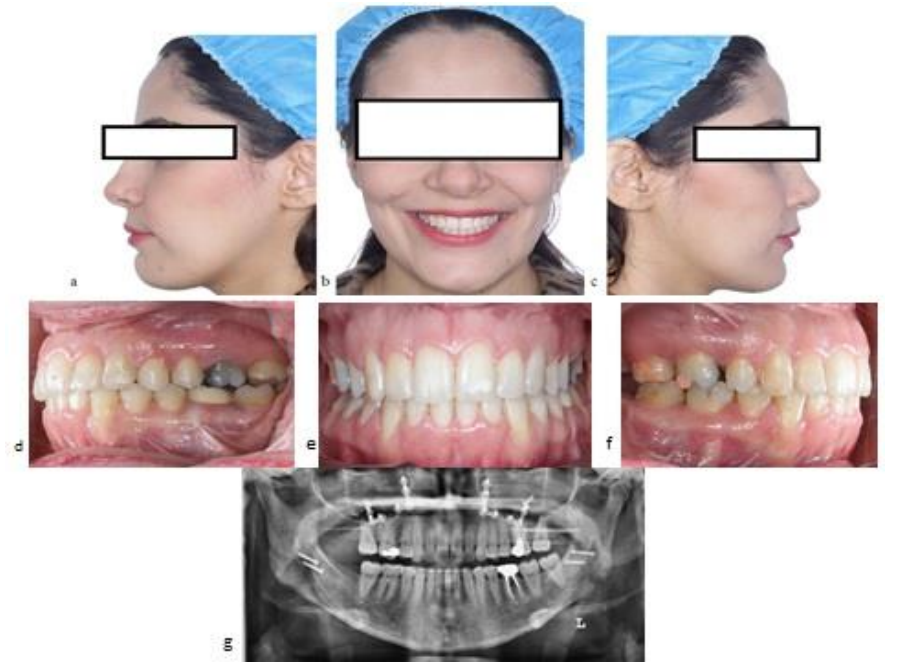

Figure 8. Post-treatment phase extraoral: (a) left, (b) frontal, (c) right; intraoral: (d) left, (e) frontal, (f) right; (g) posttreatment panoramic view. 
The maxillary roll was corrected, and normal overbite and overjet with class I molar relationship was achieved (Figure 8). Post-treatment panoramic radiograph shows root paralleling. Also, no significant evidence of root resorption was detected (Figure 8). Overbite decreased from $95 \%$ to $25 \%$ (gingival show at smile decreased from $6 \mathrm{~mm}$ to $1 \mathrm{~mm}$ ) (Figure $8)$.

Yaw correction was completely accomplished with Benefit System in the upper arch and sliding jig in the lower arch (Figure 9). There was no evidence of loss of vitality in the mandibular and maxillary teeth at the end of treatment.
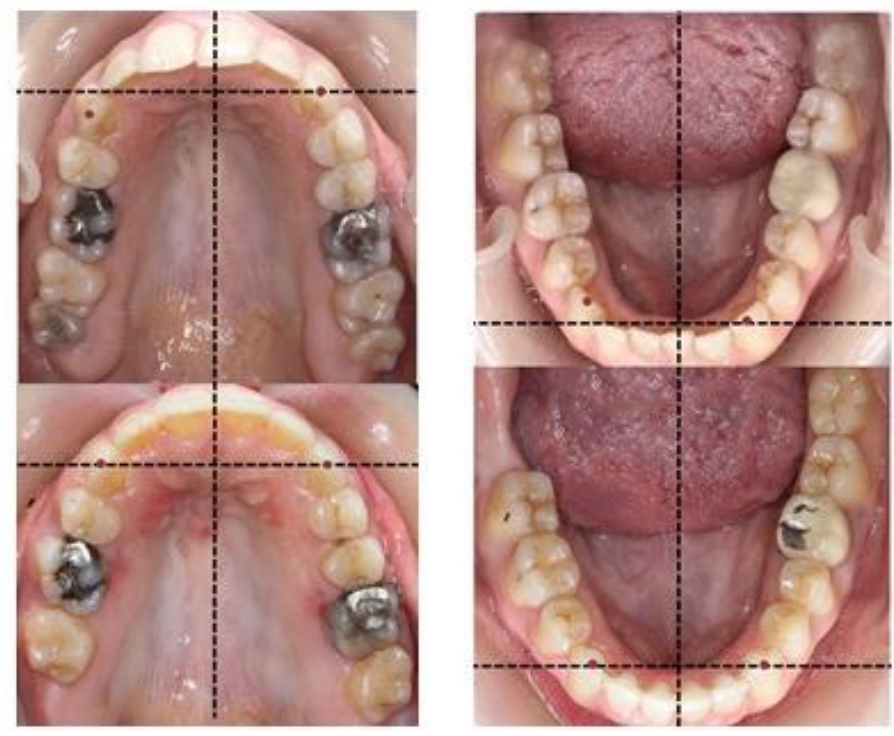

Figure 9. Yaw correction in the upper and lower arch.

\subsection{Cephalometric findings.}

The final lateral cephalometric analysis after orthognathic surgery showed skeletal cl I relationship, normal proclination of upper and lower incisor teeth (Figure 10 and Table 2), and no evidence of periodontal complication in the mandibular and maxillary teeth.

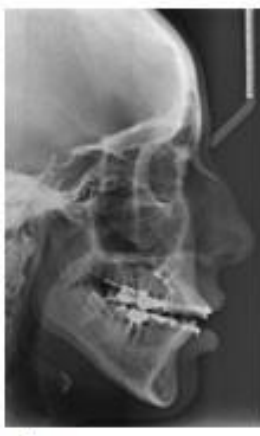

a

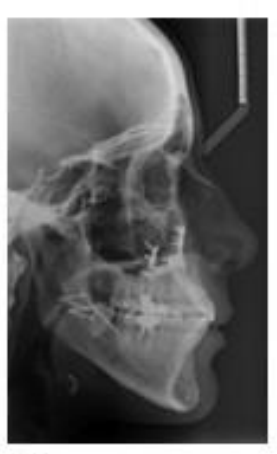

b

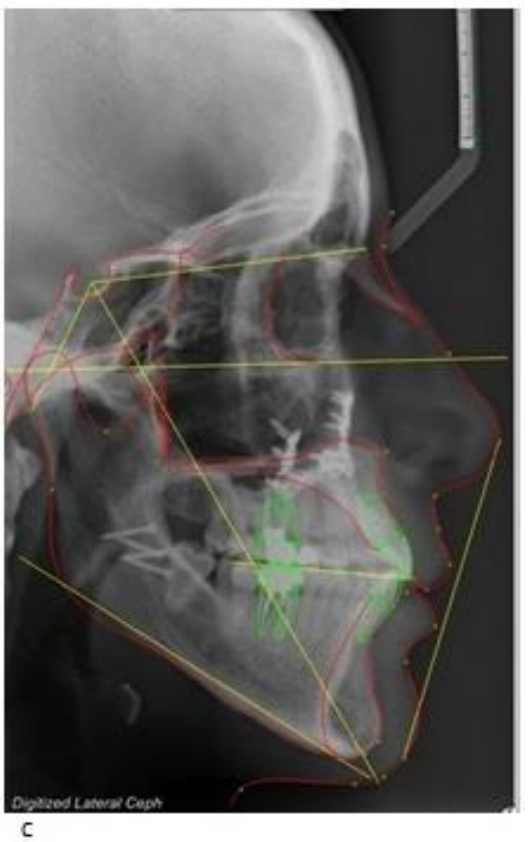

c

Figure 10. (a) Pre-surgical lateral cephalogram; (b) post-surgical lateral cephalogram; (c) digital tracing of posttreatment cephalogram. 
Table 2. Post-treatment values of cephalometric analysis.

\begin{tabular}{c|c}
$\begin{array}{c}\text { Cephalometric } \\
\text { variable }\end{array}$ & $\begin{array}{c}\text { Post Treatment } \\
\text { (degrees) }\end{array}$ \\
\hline U1-SN & 109 \\
\hline L1-MeGo & 91 \\
\hline U1 to N-Pog & 8 \\
\hline L1 to N-Pog & 6 \\
\hline Interincisal A. & 120 \\
\hline U1-Palatal P. & 112 \\
\hline S-Go: N-Mex100 & 61 \\
\hline Pal-Go-Me(Basal A.) & 37 \\
\hline SN-MeGo & 40 \\
\hline SNA & 86 \\
\hline SNB & 84 \\
\hline ANB & 2 \\
\hline SN-Pog & 84 \\
\hline Wits & -1 \\
\hline Upper lip to E_line & -2 \\
\hline Lower lip to E_line & -1 \\
\hline Nasolabial angle & 100
\end{tabular}

\subsection{Post-treatment follow up.}

After the orthodontic treatment, the patient received aesthetic restorative treatment to mask the teeth discoloration. The ideal occlusion of the patient was maintained after one year following treatment (Figure 11).
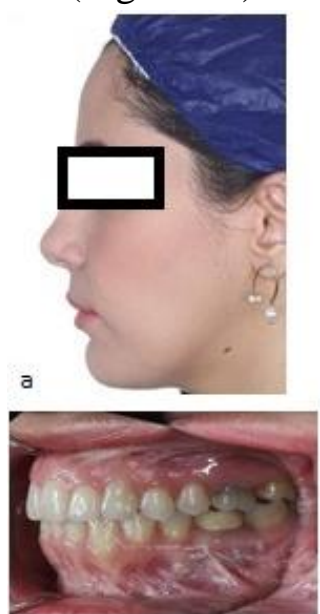
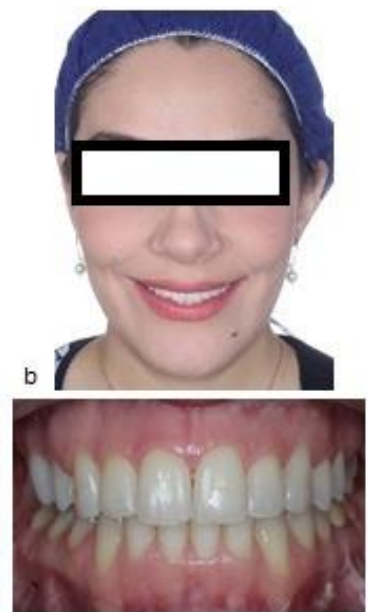

e

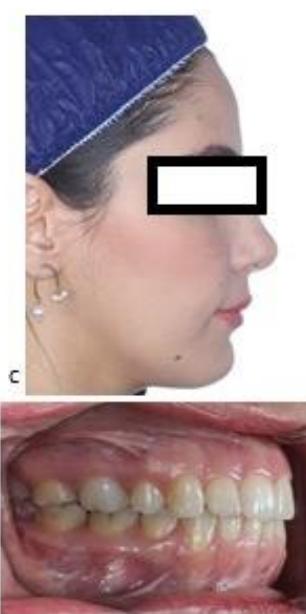

f

Figure 11. One-year follow-up phase extraoral: (a) left, (b) frontal, (c) right; intraoral: (d) left, (e) frontal, (f) right.

\subsection{Discussion.}

Total arch mesialization using TADs is an optional treatment plan for compensating an improper previous orthodontic treatment with unilateral extraction and midline deviation. Two types of absolute anchorage are proposed for this purpose; mini-screws and Bollard miniplates. The choice for mini-screws prevailed, given that they are more affordable and simple to install without the need for surgical flap repositioning [16]. In this study, we discussed unilateral total arch mesialization and also first and second molars mesialization to the space of incorrectly extracted premolar by using an innovative custom-made TAD-based jig. Root contact with mini-screws during molar mesialization is a concern during molar mesialization movement [15]. What makes this study unique is the application of an innovative technique by 
inserting only one mini-screw in a suitable area that avoids root contact and removes the need for mini screw repositioning throughout the treatment time. For maxillary and mandibular mesialization, several TADs on the buccal and palatal areas can be applied. One of these methods is to place a mini-screw in the buccal side between the roots of the canine and first premolar and using lever arm in the first molar region and applying mesialization force by the elastic chain or closed coil spring.

Another alternative is to tightly ligate the molars to the mini-screws placed mesially to the first molar as the anchorage unit and to put an open coil between the first molar and the anterior segment. After the mesialization of the anterior segment, the screw is repositioned to the anterior buccal area (for example, between the roots of first premolar and canine) for anchorage preparation in order to mesialize the posterior section. One of the limitations of this technique, according to the authors' experience is that the bone remodeling due to the mesialization of the anterior segment can interfere with the early mini-screw insertion in the anterior region because the newly remodeled bone might compromise the mini-screw stability. Therefore, the clinician might have to postpone the mini-screw repositioning until a more mature bone is created in the anterior area. In addition to the scale of tooth movement, the probability of root damage during movement progression due to close contact between the mini-screws and root and the need to reposition the mini-screws are the limitations of the miniscrew insertion between roots. Considering all these limitations, the new approach proposed in this study is utilized to overcome the aforementioned shortcomings.

Generally, mini-screw insertion in the buccal area could lead to unwanted expansion of the arch width and may cause distal out rotation in the area of the last molars since the forces line of action pass buccally to the center of resistance of the distal molars. In contrast, in the study of upper jaw mesialization by using the palatal area for mini-screw insertion, arch constriction, and tendency to the distal-in rotation of the distal molars could occur, which creates difficulties to coordinate the occlusion. To prevent these side effects, the working wire was delicately constricted in the lower arch and expanded in the upper arch in the molar area, where needed to maintain the arches' coordination throughout the treatment time. In the upper arch, adjustment of the Beneplate (making buccal expansion as the molar moves mesially) also helped in preserving the upper arch form. Although, the patient's tight occlusion also assisted in the arch coordination maintenance.

In this study mesialization of the lower posterior segment was successfully conducted by a novel TAD-based jig in a reasonable time and without any root resorption or adverse effect on associated surrounded alveolar ridge or periodontal structures. Interestingly, throughout the mesialization phase, we noticed that the speed of teeth mesialization was higher in the lower arch (using custom-made jig) than the upper arch (using the Benefits system). This result is in line with the result of a study reported that the lower molar mesialization using TADs diminished treatment duration and supported more prognostic tooth movement without compromising associated bone level and periodontal structures [17]. It is also reported that extensive molar mesialization up to $15 \mathrm{~mm}$ is predictably possible with the aid of absolute anchorage [18]. This result is in line with our study, although the aforementioned study was conducted on a growing patient, whereas we discussed molar movement on an adult woman.

In this patient, no clinical considerable apical root resorption occurred, which is in line with the results reported by Wrinkler et al. that claimed that space closure through mesialization is a safe treatment option in terms of apical root resorption [1]. In this research, no unwanted tooth tipping or angulation alteration occurred, which is one of the advantages of 
the discussed innovative jig and its favorable force line for mandibular arch mesialization. In this study, the location of the loop (distally to the second premolar) and the length of the loop was designed by considering the center of rotation of the moving segment so that the point of the force application was in the line of the center of the rotation of the posterior segment. As reported recently, careful selection of force angulation and biomechanics is crucial to accomplish proper tooth movement in total mesialization of the mandibular dentition [19]. In the current study, we agreed with other studies that concluded that the mesialization of the posterior segment should be conducted with rectangular archwires that fully fit the slot for torque expression, rotation, and anchorage to prevent undesirable movements [20, 21]. Although, a study reports the successful and stable mesial movement of the maxillary second and third molars was obtained without bracket bonding on the anterior dentition with the use of an implant-supported appliance (Mesialslider) [22]. We exerted mesial force for total arch mesialization on the $19 \times 25$ " stainless steel working archwire to have three-dimensional control on the teeth positions.

\section{Conclusions}

Incorrect unilateral extraction of teeth is one of the challenging issues in orthodontics. The unilateral mesialization approach used in the current study prevented invasive therapies such as dental implants employing a special custom-made TAD-based jig, which is less aggressive and more durable for the patient and revealed successful results with minimum side effects. Also, this approach eliminated the need for patient cooperation for elastic wear for arch mesialization, which could cause complications such as occlusal plane cant or associated teeth rotation.

\section{Funding}

There is no financial support.

\section{Acknowledgments}

The authors would like to thank the Department of Orthodontics, School of dentistry, Shahid Beheshti, and Islamic Azad University of Medical Sciences, Tehran, Iran.

\section{Conflicts of Interest}

The authors declare no conflict of interest.

\section{References}

1. Winkler, J.; Göllner, N.; Göllner, P.; Pazera, P.; Gkantidis, N. Apical root resorption due to mandibular first molar mesialization: A split-mouth study. Am J Orthod Dentofac 2017, 151, 708-717, https://doi.org/10.1016/j.ajodo.2016.12.005.

2. Klang, E.; Beyling, F.; Knösel, M.; Wiechmann, D. Quality of occlusal outcome following space closure in cases of lower second premolar aplasia using lingual orthodontic molar mesialization without maxillary counterbalancing extraction. Head Face Med 2018, 14, 17, https://doi.org/10.1186/s13005-018-0176-2.

3. Deguchi, T.; Nasu, M.; Murakami, K.; Yabuuchi, T.; Kamioka, H.; Takano-Yamamoto, T. Quantitative evaluation of cortical bone thickness with computed tomographic scanning for orthodontic implants. Am $J$ Orthod Dentofacial Orthop 2006, 129, 721 e7-12, https://doi.org/10.1016/j.ajodo.2006.02.026.

4. Karami, A.; Tebyanian, H.; Barkhordari, A.; Motavallian, E.; Soufdoost, R.S.; Nourani, M.R. Healing effects of ointment drug on full-thickness wound. C. R. Acad. Bulg. Sci 2019, 72, 123-129, https://doi.org/10.7546/CRABS.2019.01.16. 
5. Shakeri, F.; Tebyanian, H.; Karami, A.; Babavalian, H.; Tahmasbi, M.H. Effect of Topical Phenytoin on Wound Healing. Traum Month 2016, Inpress, e35488, https://doi.org/10.5812/traumamon.35488.

6. Soufdoost, R.S.; Mosaddad, S.A.; Salari, Y.; Yazdanian, M.; Tebyanian, H.; Tahmasebi, E.; Yazdanian, A.; Karami, A.; Barkhordari, A. Surgical Suture Assembled with Tadalafil/Polycaprolactone Drug-Delivery for Vascular Stimulation Around Wound: Validated in a Preclinical Model. Biointerface Res. Appl. Chem 2020, 10, 6317-6327, https://doi.org/10.33263/briac105.63176327.

7. Tahmasebi, E.; Alikhani, M.; Yazdanian, A.; Yazdanian, M.; Tebyanian, H.; Seifalian, A. The current markers of cancer stem cell in oral cancers. Life Sci 2020, 249, 117483, https://doi.org/10.1016/j.lfs.2020.117483.

8. Kafshgari, H.S.; Yazdanian, M.; Ranjbar, R.; Tahmasebi, E.; Mirsaeed, S.R.G.; Tebyanian, H.; Ebrahimzadeh, M.A.; Goli, H.R. The effect of Citrullus colocynthis extracts on Streptococcus mutans, Candida albicans, normal gingival fibroblast and breast cancer cells. Journal of Biological ResearchBollettino della Società Italiana di Biologia Sperimentale 2019, 92, https://doi.org/10.4081/jbr.2019.8201.

9. Mosaddad, S.A.; Tahmasebi, E.; Yazdanian, A.; Rezvani, M.B.; Seifalian, A.; Yazdanian, M.; Tebyanian, H. Oral microbial biofilms: an update. Eur J Clin Microbiol Infect Dis 2019, 38, 2005-2019, https://doi.org/10.1007/s10096-019-03641-9.

10. Khomarlou, N.; Aberoomand-Azar, P.; Lashgari, A.P.; Tebyanian, H.; Hakakian, A.; Ranjbar, R.; Ayatollahi, S.A. Essential oil composition and in vitro antibacterial activity of Chenopodium album subsp. striatum. Acta Biol Hung 2018, 69, 144-155, https://doi.org/10.1556/018.69.2018.2.4.

11. Arbuckle, G.; Nelson, C.; Roberts, W. Osseointegrated implants and orthodontics. Oral Maxillofac Surg Clin North Am 1991, 3, 903.

12. Atarod, M.; Safari, J.; Tebyanian, H. Ultrasound irradiation and green synthesized CuO-NiO-ZnO mixed metal oxide: An efficient sono/nano-catalytic system toward a regioselective synthesis of 1-aryl-5-amino1H-tetrazoles. Synthetic Commun 2020, 50, 1993-2006, https://doi.org/10.1080/00397911.2020.1761396.

13. Soufdoost, R.S.; Yazdanian, M.; Tahmasebi, E.; Yazdanian, A.; Tebyanian, H.; Karami, A.; Nourani, M.R.; Panahi, Y. In vitro and in vivo evaluation of novel Tadalafil/ $\beta$-TCP/Collagen scaffold for bone regeneration: A rabbit critical-size calvarial defect study. Biocybern Biomed Eng 2019, 39, 789-796, https://doi.org/10.1016/j.bbe.2019.07.003.

14. Karami, A.; Tebyanian, H.; Goodarzi, V.; Shiri, S. Planarians: an In Vivo Model for Regenerative Medicine. Int J Stem Cells 2015, 8, 128-33, https://doi.org/10.15283/ijsc.2015.8.2.128.

15. Baik, U.; Kook, Y.-A.; Tanaka, O.M.; Kim, K.B. Root contact with miniscrews during mesiodistal movement of the molar. J. World Fed. Orthod 2014, 3, e95-e100, https://doi.org/10.1016/j.ejwf.2014.02.001.

16. De, H.C.; Cornelis, M.A. Biomechanics of skeletal anchorage. Part 2: Class II nonextraction treatment. $J$ Clin Orthod 2006, 40, 290-8; quiz 307.

17. Rivis, O.; Potapchuk, A.; Goncharuk-Khomyn, M.; Bokoch, A. Use of Mini-Implant Anchorage For Second Molar Mesialization: Comprehensive Approach For Treatment Efficiency Analysis. Pesqui. Bras. Odontopediatria Clín. Integr 2019, 20, 5262, https://doi.org/10.1590/pboci.2020.018.

18. Rédua, R.B.; Rédua, P.C.B.; Ferreira, C.E.d.A.; Ortega, A.d.O.L. Orthodontic approach to treat complex hypodontia using miniscrews in a growing patient. Dental Press J Orthod 2015, 20, 82-90, https://doi.org/10.1590/2176-9451.20.4.082-090.oar.

19. Kawamura, J.; Park, J.H.; Kojima, Y.; Kook, Y.A.; Kyung, H.M.; Chae, J.M. Biomechanical analysis for total mesialization of the mandibular dentition: A finite element study. Orthod Craniofac Res 2019, 22, 329336, https://doi.org/10.1111/ocr.12331.

20. Sandler, P.J.; Atkinson, R.; Murray, A.M. For four sixes. Am J Orthod Dentofacial Orthop 2000, 117, 41834, https://doi.org/10.1016/s0889-5406(00)70161-2.

21. Carrera Garrido, E.; Sáez Espínola, G. Compensation due to lower first molar absence by means of traditional unilateral mesialization of the posterior segment. Rev. Mex. de Ortod 2016, 4, 121-126, https://doi.org/10.1016/j.rmo.2016.10.016.

22. Wilmes, B.; Vasudavan, S.; Drescher, D. Maxillary molar mesialization with the use of palatal mini-implants for direct anchorage in an adolescent patient. Am J Orthod Dentofacial Orthop 2019, 155, 725-732, https://doi.org/10.1016/j.ajodo.2019.01.011. 